\title{
Multiple Pigmented Seborrheic Keratosis with Sebaceous Differentiation - A Case Report
}

\author{
R Vimal CHANDER, P JAYAGANESH, Ganthimathy SEKHAR, S CHITRA
}

Department of Pathology, Saveetha Medical College and Hospital, CHENNAI, INDIA

\begin{abstract}
Seborrheic keratosis is a benign lesion that is common in the trunk and head and neck regions. It shows a considerable variety of histological appearances leading to different variants. The presence of sebaceous differentiation in seborrheic keratosis is very rare and can histologically mimic benign and malignant tumors with sebaceous differentiation. We present a case of a 65 -year-old male presenting with multiple nodules over the right and left sides of neck and the right preauricular region, histopathological examination of which revealed multiple pigmented seborrheic keratoses with sebaceous differentiation. This case is reported for its rare sebaceous differentiation and multiplicity along with the importance of differentiating it from a variety of benign to malignant neoplasms showing sebaceous differentiation.
\end{abstract}

Key Words: Seborrheic keratosis, Sebaceous differentiation, Differential diagnosis

\section{INTRODUCTION}

Seborrheic keratosis is a benign epidermal lesion occurring mainly on the trunk, head and neck region and in the extremities, excluding palms and soles, usually after the age of 50 years (1). They are sharply demarcated, brownish slightly raised from the surface with a stuck-on appearance. Seborrheic keratoses show a considerable variety of histological appearances including the irritated type, adenoid or reticulated type, clonal type, melanoacanthoma type, inverted follicular keratosis and benign squamous keratosis types. All types show hyperkeratosis, acanthosis and papillomatosis. Thepresence of sebaceous differentiation in seborrheic keratosis is very rare. We present a case of multiple seborrheic keratosis with sebaceous differentiation in a 65 year old male.

\section{CASE REPORT}

A 65-year-old male presented with multiple wart-like lesions over the right and left sides of the neck and in the right preauricular region for the past 3 years. An excisional biopsy was performed and sent for histopathological examination.

Macroscopically, there were multiple skin-covered irregular gray brown soft tissue fragments varying in size from $1 \mathrm{~cm}$ in diameter to $2 \times 2 \times 1 \mathrm{~cm}$, with cut section showing black and yellow areas (Figure 1).

(Turk Patoloji Derg 2018, 34:108-111)

Received : 08.01.2015 Accepted : 24.02.2015
Microscopically, the section showed hyperplastic stratified squamous epithelium with acanthosis and proliferation of basaloid cells, papillomatosis and pigment deposition throughout. Multiple interspersed horn and pseudohorn cysts are seen (Figure 2). Deeper areas of the lesion showed small clusters and scattered sebaceous cells intermixed with the basaloid cells (Figures 3,4). There was no evidence of atypia or increased mitotic figures thus ruling out malignancy.

A final diagnosis of pigmented seborrheic keratosis with sebaceous differentiation was made. His complete blood count, serum urea and electrolytes, liver function tests and serum protein electrophoresis was found to be within normal limits. Chest X-ray, abdominal computed tomography, upper gastrointestinal endoscopy and colonoscopy were also performed which did not reveal any internal malignancy. No mass or recurrence was noted during the routine follow up after 6 months.

\section{DISCUSSION}

Seborrheic keratosis is a benign epidermal lesion occurring mainly on the trunk, commonly in the interscapular area, sides of the neck, face and also on the extremities, excluding palms and soles, usually after the age of 50 years (1). They are sharply demarcated, brownish with a verrucous surface, slightly raised from the surface. The lesions are coin-like, sharply demarcated from the surrounding skin with a stuck-on appearance (2). Rarely, they can have a targetoid appearance (3).

Correspondence: R. Vimal CHANDER

Department of Pathology, Saveetha Medical College,

Thandalam, 602105, Chennai, INDIA

E-mail: rvimalchander@gmail.com Phone: +9790645729 


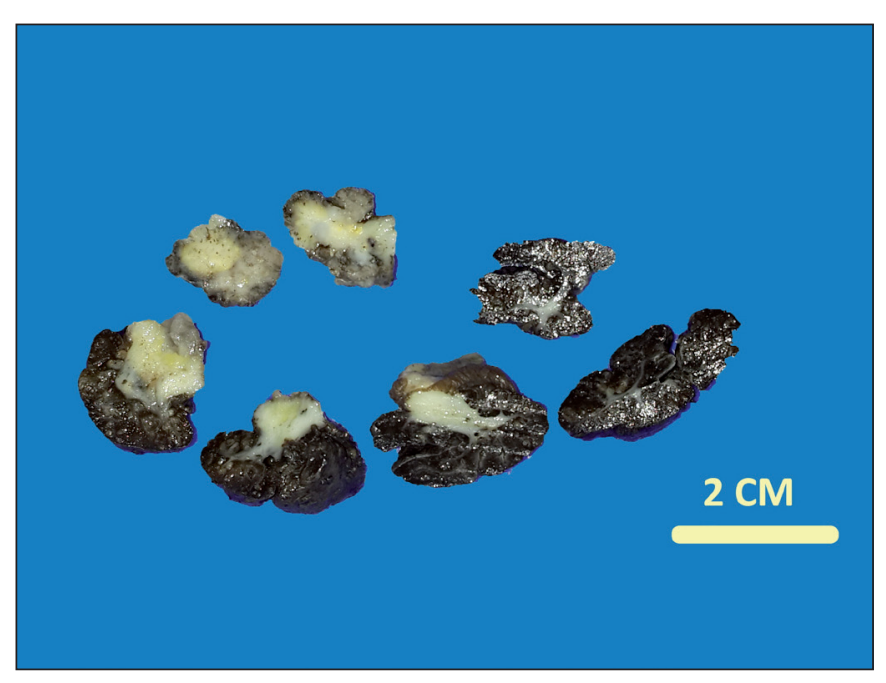

Figure 1: Gross appearance of the lesion

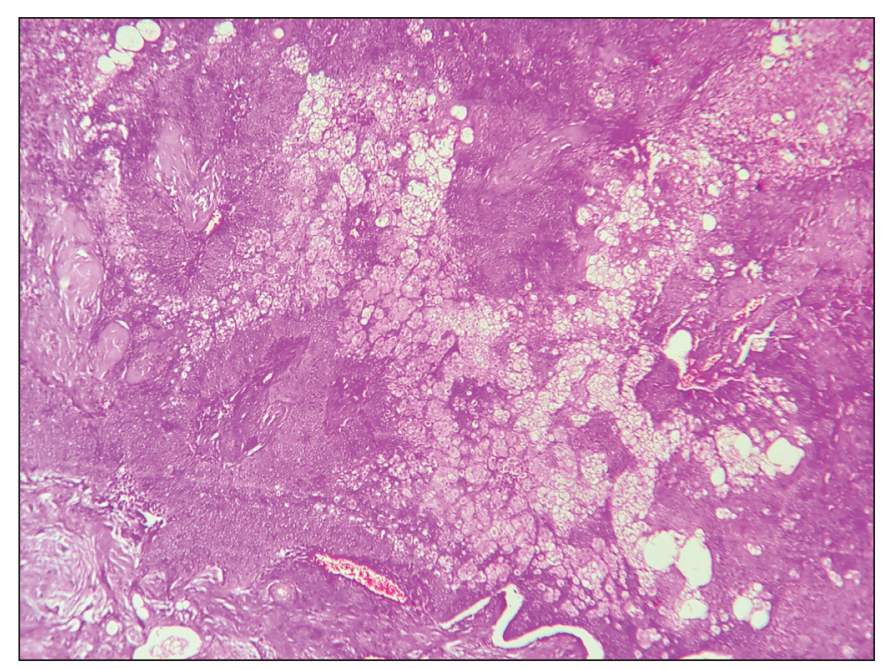

Figure 3: Seborrheic keratosis with deeper areas showing sebaceous differentiation (H\&E; $\mathrm{x} 40)$.

The lesions are composed of basaloid cells with an admixture of varying amount of squamoid cells. Keratin-filled invaginations and small cysts (horn cysts) are a common feature. Approximately one third of the lesions appear hyperpigmented. Seborrheic keratoses show a considerable variety of histological appearances and is usually classified into several subtypes: acanthotic, hyperkeratotic, adenoid or reticulated, clonal, irritated, desmoplastic, inverted follicular keratosis and melanoacanthoma. All types show hyperkeratosis, acanthosis and papillomatosis (4). Rare changes include focal trichilemmal differentiation with glycogen-rich cells, acantholysis with basal clear cells and sebaceous differentiation. Very rarely, seborrheic keratosis

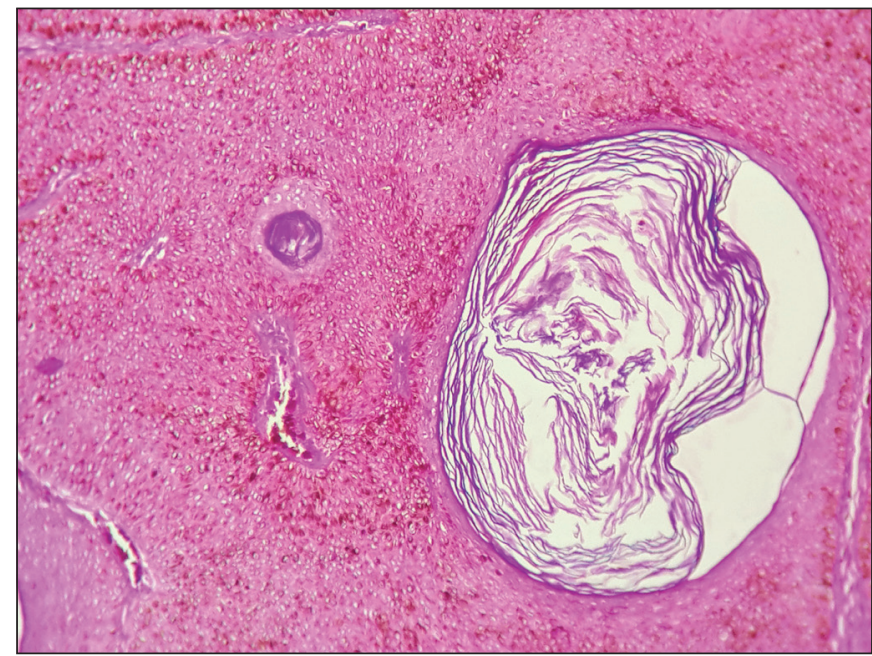

Figure 2: Microscopy showing acanthosis, proliferation of basaloid cells with melanin pigments in the cytoplasm along with a horn cyst (H\&E; x100).

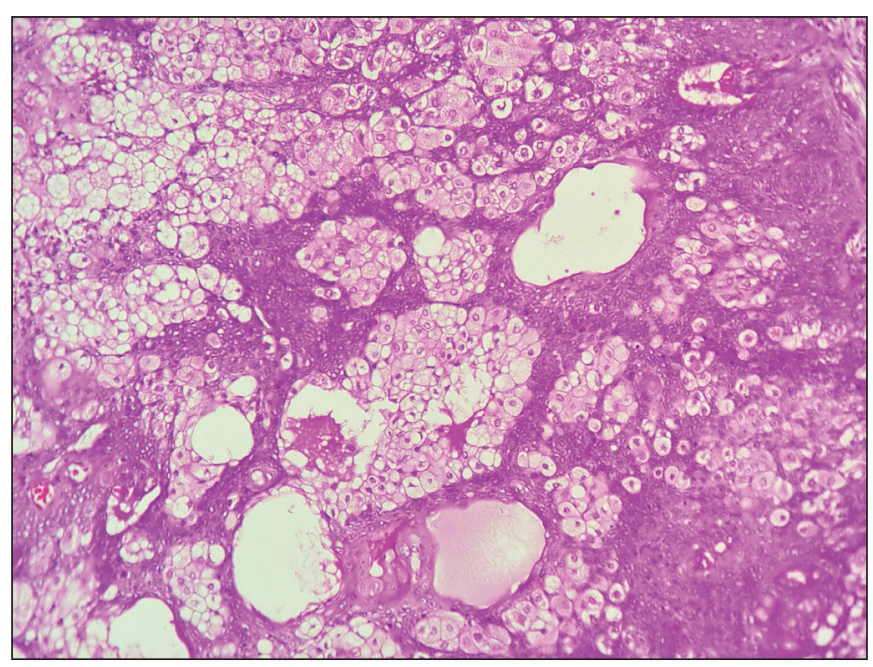

Figure 4: Areas showing sebaceous differentiation amongst the basaloid cells (H\&E; x100).

with pseudorosettes and adamantinoid types has also been reported as two new histologic variants (5).

The differential diagnosis of seborrheic keratosis ranges from various benign lesions such as papilloma, senile keratosis, keratoacanthoma and verrucous epidermal hyperplasia to malignant lesions such as basal or squamous cell carcinoma (6). Very rarely, progressive transformation to in-situ squamous cell carcinoma (bowenoid transformation) has been reported (7).

The presence of sebaceous differentiation in seborrheic keratosis is very rare. The differential diagnosis of such cases include sebaceoma variant with surface changes of verruca/ 
seborrheic keratosis, and other tumors with sebaceous differentiation such as trichoblastoma with sebaceous differentiation, sebocrine adenoma (apocrine poroma with sebaceous differentiation), superficial epithelioma with sebaceous differentiation, low-grade sebaceous carcinoma and basal cell carcinoma with sebaceous differentiation.

In the sebaceoma variant with surface changes of verruca/ seborrheic keratosis, there is proliferation of infundibular keratinocytes (basaloid cells or squamous cells) associated with hypergranulosis and tunnels of cornified cells (thus mimicking verruca or seborrheic keratosis) with a random admixture of sebaceous cells. In contrast to seborrheic keratosis, sebaceoma is centered on the upper and mid dermis, with an increased proportion of sebaceous cells compared to the basal cells (8). Recently, the term "sebomatricoma" has been proposed as the most appropriate term for all benign neoplasms with sebaceous differentiation including seborrheic keratosis with sebaceous differentiation $(9,10)$.

Rarely, sebaceoma may arise in continuity with seborrheic keratosis wherein the lesion may be composed of two different adjacent tumors - seborrheic keratosis with hyperkeratosis and acanthosis with irregular proliferation of apparently benign basaloid and squamous keratinocytes and small horn pseudocysts and the other part composed of sebaceoma characterised by various sized lobules with immature sebocytes mixed with single or clustered mature sebaceous cells (11).

In trichoblastoma with sebaceous differentiation, there is proliferation of follicular germinative cells associated with a highly fibrotic stroma and peripheral palisading in contrast to seborrheic keratosis. Sebocytes and sebaceous duct like structures are observed within the basaloid aggregations (8).

In contrast to seborrheic keratosis, apocrine poroma with sebaceous differentiation (sebocrine adenoma) is a benign cutaneous adnexal neoplasm differentiating in the direction of sebaceous and apocrine glands. The sebaceous differentiation is characterized by solitary instances or clusters of sebocytes and sebaceous ducts. The apocrine differentiation is characterized by basaloid aggregations composed of poroid cells and cuticular cells associated with tubular structures (12).

Superficial epithelioma with sebaceous differentiation is a rare histologically distinct benign neoplasm, occurring as multiple or solitary lesions on the face, neck and trunk in elderly persons. Histologically it shows superficial well- demarcated plate like proliferation of basaloid cells with numerous broad attachments to the overlying epidermis with gradual merging of the tumor cells with the overlying epidermal cells at the dermoepidermal junction. Sebaceous cells scattered as small clusters may be seen in the lower portion of these lobules. Within the tumor mass, few keratin filled cystic structures lined with squamous epithelium of epidermal or sebaceous duct. There is no peripheral palisading of the cells nor any retraction artefact (13).

Low-grade sebaceous carcinoma is composed of cytologically neoplastic cells with large, crowded, vesicular pleomorphic nuclei with prominent nucleoli, which is absent in seborrheic keratosis. Rarely some cases of basal cell carcinoma present with areas of sebaceous differentiation, which can be differentiated from seborrheic keratosis by the characterictic architectural pattern with peripheral palisading and cytology of the neoplastic cells in basal cell carcinoma (8).

In the present case, there was hyperplastic stratified squamous epithelium with acanthosis and proliferation of uniform basaloid cells with interspersed horn and pseudohorn cysts with deeper areas showing sebaceous differentiation. The proportion of the sebaceous cells are lower compared to the basaloid cells, in contrast to sebaceoma. There was absence of peripheral palisading, fibrotic stroma, duct-like structures or platelike proliferation of basaloid cells. Thus a diagnosis of seborrheic keratosis with sebaceous differentiation is arrived at.

Although seborrheickeratosis with sebaceous differentiation is not known to present with the rare Muir-Torre syndrome, any benign sebaceous or transitional squamosebaceous neoplasm should be considered a possible manifestation of this syndrome (14).

Complete surgical excision is the curative method although local destructive methods such as cryotherapy or curettage can also be done.

Sudden onset of numerous seborrheic keratosis (termes as Leser-Trelat sign) has been reported in most of the cases in association with internal malignancy, most commonly adenocarcinoma of the stomach (15). Other associated tumors include leukemia, bronchogenic carcinoma, breast carcinoma, colorectal carcinoma, renal cell carcinoma, transitional cell carcinoma and anaplastic ependymoma. In contrast, occasional cases have been reported with the Leser-Trelat sign without internal malignancy even after extensive investigations (16). 
Patients with multiple seborrheic keratosis are at risk for internal malignancy. Hence these patients need to be under routine screening and regular follow up.

In conclusion, seborrheic keratosis is a benign epidermal lesion characterised by hyperkeratosis, acanthosis along with keratin-filled horn cysts and pseudohorn cysts, and various subtypes have been reported. Sebaceous differentiation in seborrheic keratosis, as in the present case, is very rare. This case is reported for its rare sebaceous differentiation and multiplicity along with the importance of differentiating it from a variety of benign to malignant neoplasms showing sebaceous differentiation.

\section{REFERENCES}

1. Zhang RZ, Zhu WY. Seborrheic keratoses in five elderly patients: An appearance of raindrops and streams. Indian J Dermatol. 2011;56:432-4.

2. Bhuiyan ZH. Seborrheic Keratosis: A case report. ORION Medical Journal. 2007; 26:441-2.

3. Yoon NY, Kim BK, Hong SP, Jeon SY, Ahn SK. Cockarde (targetlike lesion) seborrheic keratosis: An unusual clinical pattern. Ann Dermatol. 2013;25:512-4.

4. Hafner C, Vogt T. Seborrheic keratosis. J Dtsch Dermatol Ges. 2008;6:664-77.

5. Requena L, Kutzner H. Seborrheic keratosis with pseudorosettes and adamantinoid seborrheic keratosis: Two new histopathologic variants. J Cutan Pathol. 2006;33 Suppl 2:42-5.

6. Rao BK, Freeman RG, Poulos EG, Arbesfeld L, Rendon M. The relationship between basal cell epithelioma and seborrheic keratosis. A study of 60 cases. J Dermatol Surg Oncol. 1994;20:761-
7. Rajabi P, Adibi N, Nematollahi P, Heidarpour M, Eftekhari M, Siadat AM. Bowenoid transformation in seborrheic keratosis: A retrospective analysis of 429 patients. J Res Med Sci. 2012;17:21721.

8. Misago N, Mihara I, Ansai S, Narisawa Y. Sebaceoma and related neoplasms with sebaceous differentiation: A clinicopathologic study of 30 cases. Am J Dermatopathol. 2002;24:294-304.

9. Sáchez Yus E, Requena L, Simón P, del Río E. Sebomatricoma: A unifying term that encompasses all benign neoplasms with sebaceous differentiation. Am J Dermatopathol. 1995;17:213-21.

10. Requena L, Kuztner H, Fariña MC. Pigmented and nested sebomatricoma or seborrheic keratosis with sebaceous differentiation? Am J Dermatopathol. 1998;20:383-8.

11. Betti R, Inselvini E, Vergani R, Moneghini L, Crosti C. Sebaceoma arising in association with seborrheic keratosis. Am J Dermatopathol. 2001;23:58-61.

12. Lee NH, Lee SH, Ahn SK. Apocrine poroma with sebaceous differentiation. Am J Dermatopathol. 2000;22:261-3.

13. Lee MJ, Kim YC, Lew W. A case of superficial epithelioma with sebaceous differentiation. Yonsei Med J. 2003;44:347-50.

14. Jakobiec FA, Zimmerman LE, La Piana F, Hornblass A, Breffeilh RA, Lackey JK. Unusual eyelid tumors with sebaceous differentiation in the Muir-Torre syndrome. Rapid clinical regrowth and frank squamous transformation after biopsy. Ophthalmology. 1988;95:1543-8.

15. Venegas FA, Vaccaro PM, Abudinén GA, Reydet CV, Brunie FV, Arcuch JD. Leser-Trélat sign associated with gastric cancer: Report of one case. Rev Med Chil. 2012;140:1585-8.

16. Safa G, Darrieux L. Leser-Trélat sign without internal malignancy. Case Rep Oncol. 2011;4:175-7. 